\title{
On the psychology of mathematical problem solving by gifted students
}

\author{
SANDOR M. VERES
}

Dedicated to the Memory of Zoltán Szvetits

\begin{abstract}
This paper examines the nature of mathematical problem solving from a psychological viewpoint as a sequence of mental steps. The scope is limited to solution processes for well defined problems, for instance, which occur at International Mathematical Olympiads. First the meta-mathematical background is outlined in order to present problem solving as a well defined search problem and hence as a discovery process. Solving problems is described as a sequence of elementary steps of the so called "relationship-vision" introduced here. Finally, non-procedural aspects of the psychology of problem solving are summarized, such as the role of persistence, teacher-pupil relationship, the amount of experience needed, self-confidence and inspiration at competitions.
\end{abstract}

Key words and phrases: methodology and psychology of mathematical problem solving, competitions, inspiration, intuition, heuristics, teaching mathematics, gifted pupils.

ZDM Subject Classification: D50, C30.

\section{Introduction}

As one of the seminal papers [18] points out, mathematical problem solving can mean different things to different professionals, depending on the activity they pursue. To an engineer it can mean the building of physical or data-based models of a static or dynamic nature, which then they use to solve an engineering problem. Mathematical models are used for instance for design optimisation or development of electronic feedback controllers. To pupils at school, it primarily means solving well defined mathematical problems in the classroom or at competitions. 
The problems to be solved may not be well defined to developers of mathematics whose task is to discover new relationships and to prove new and interesting theorems. This process is similar to developers of control sciences [21], where theorems are developed and proved about new mathematical structures invented for controlling physical dynamics and also for the development of artificial intelligence.

To pure mathematicians, problem solving can sometimes also mean work devoted to proving or disproving a conjecture of a colleague. Furthermore, problem solving can have a different meaning for a medical and social scientist or economist, who would like to use data and statistics to discover new relationships.

On the other hand, the psychology of problem solving is mainly concerned with the nature of the conscious, as well as the subconscious, mental processes during problem solving. This can include conscious control of the discovery process as well as subconscious associative processes of the brain supported by memories of problem solving experience. The discovery process is shared with developmental mathematics, though the description of the psychology is narrower in this paper than, for instance, in the collection of papers [6]. In his essay, Hadamard [7] describes a rich set of observations on the role of subconscious, aesthetics, choices made in the discovery process of mathematical theories and the workings of a variety of mathematical minds.

Our main concern in this paper is mathematical problem solving of well defined problems in general, with particular focus on competitions of able pupils. We will attempt some objective analysis, which is inevitably influenced by subjective introspection of the author, and observation of some pupils at work.

We make the following assumptions:

(1) There is a well defined mathematical problem $P$, which is the subject of problem solving, of the following types:

(a) some relationship $R$ needs to be proved;

(b) existence or non-existence of some mathematical models $M$ or relationships $R$ are to be proved;

(c) all solutions, $S$ satisfying some relationship $R$, are to be found.

(2) The person $H$ attempting to solve problem $P$ is familiar with all the mathematical concepts in which problem $P$ is stated.

(3) The person $H$ has some experience $E$ in solving problems similar to $P$.

For instance, proving a relationship can be of any kind: geometric, equalities, inequalities, number theoretic, graph theoretic, combinatorial, etc. These share 
a common feature in that a set of relationships are given and the problem solver needs to derive a goal one. The problem of proving the existence of some mathematical models or relationships, or the lack of them, is somewhat different in its character, as in this case the task is not to "find a pathway of derivation" but to find any means that under some condition something must exist or otherwise. Finding all models or relationships, which satisfy some given criteria, is also very general: the entries can be numbers, sets of numbers, series, geometric shapes, combinatorial constructs of any kind.

We will attempt to provide some formal descriptions of the mental operations involved in problem solving, without completeness proved. Psychology includes however more: mental attitudes such as self confidence, persistence, mental capacity, relevance of associations and judgements or self-confidence in one's ability.

\section{Meta-mathematics of problem solving}

Although this paper is concerned with the human condition of problem solving in terms of psychological concepts, we provide a brief overview of the "mathematics of problem solving", which is often called meta-mathematics.

Most problems at competitions of secondary school pupils can be stated within some area of mathematical theory which is completely determined by a finite set of axioms. Geometry is finitely axiomatic [16], the Peano axioms make number theory also axiomatic, set theory with Zermelo-Fraenkel axioms, plus the axiom of choice (ZFC,[9]), make combinatorics and the calculus axiomatic [8].

Like a flowing river of inference, it is the inference-flow of first and higher order logic [17], which enables most of mathematics to be treated with the rigour of axiomatic logic [13], using predicates and first and higher order logic with existential $(\exists)$ and universal $(\forall)$ quantifiers. By poetic analogy, the wells are the assumptions based on axioms and the streams and rivers are various mathematical theories, which sometimes meet and mix. Application of first and higher order logic to mathematical assumptions $[17,13])$ enables automated reasoning on computers.

It is also well known, by Gödel's theorem, that there is no generic algorithm to solve all problems [17]. Limited results on automated provability within subtheories are obtained every year.

Heuristics in problem solving involves mental short-cuts that ease the cognitive load of making a decision. Examples of this method include using an 
educated guess, an intuitive judgement or common sense, given the estimated degree of complexity of the problem.

Principles of heuristics are gradually applied to theorem proving. Among the first was ADEPT [1], a basically heuristic theorem-prover for group theory, which incorporated many of the techniques of the human mathematician in a "natural" way. ADEPT has proved nearly a hundred theorems. Some early computer-based heuristic theorem-proving results can be found in $[4,12]$. [5] presents a theoremproving system based on interaction between man and machine. The book [11] defined and developed heuristics as intelligent search strategies for computer-based problem solving. The special issue [3] contains some generic theorem-proving results on problems presentable in first order predicate logic. The International Joint Conference of Automated Reasoning series - IJCAR [2]) collects some of the ongoing new results in the area of automated problem solving in mathematics.

In fact nearly all of the problems set at competitions could now be placed into theorem-provers to find their solutions but without guaranteed or quick results. Some would protest that "the fun of human problem solving" can be lost if we were to make machines solve problems. This is clearly an unfounded worry as human sports continue to develop today, despite that we created powerful machines to replace our muscle power. But we are not there yet. The amazingly rich modelling and association capabilities of the human brain still generally exceed those of artificial intelligence machines. This may not remain so for ever, but appears that new mathematics, and the best of mathematics today, is still being developed by humans.

For the psychology of human problem solving, using our brains, it is good to be aware of the nature of problem solving and that all solutions could potentially be developed from a finite set of axioms, and assumptions, using mathematical logic. It is a discovery process rather than anything else. Knowing this classifies mathematical problems as search problems under completely well defined conditions, where skills in search methods and heuristics are important.

The challenge of problem solving by the human brain is therefore: how to handle mathematical relationships across mental maps. Effectiveness of this process fundamentally depends on prior knowledge of former mathematical results, techniques and on the ability of judging what could work for a solution. 


\section{The psychology of problem solving}

General theories of problem solving have been investigated in psychology. An introduction can be found in [10]. In psychology, problem solving is studied as a cognitive process of the human brain [20]. Problem solving is defined as a process of the cortex that searches for a solution by finding a path to reach a goal. General traits of problem solving have been identified in psychology: for instance finding a direct solution path using known solutions. Analogy is used to reduce a new problem to an existing or similar one for which solutions have already been known. Heuristics can be used to guess and adopt the "most possible" solutions. Hill climbing means to make any move that approaches the solution ever closer, step by step. Exhaustive search is used in systematic search for all possible solutions, mainly by breadth-first or depth-first strategies. Divide-andconquer is the principle of solving a whole problem via decomposing it into a set of sub-problems. Analysis and synthesis means reducing a problem to relationships across a mental map so that it becomes more suitable for the discovery of overall relationships. More details on the cognitive theory of problem solving can be found in [20].

\subsection{Polya's principles}

In the specific area of problem solving in mathematics, George Polya's work is perhaps the most widely known today. His theory is clearly formulated from the viewpoint of a mathematician but is in fact fundamentally psychological as it does not attempt to create a mathematical theory of problem solving. In [14], and later in [15], Polya presented his four-step problem solving method, which can be summarised as follows.

(1) Understand the problem: this is a "cognitive check" for the completeness of conceptual prerequisites of understanding. A teacher can help this process by a series of questions, for instance "is there enough information to enable you to find a solution?".

(2) Devise a plan: This step is about choosing an appropriate strategy to attack the problem. Some examples are: draw a picture, use a formula, guess and check, make an orderly list, eliminate possibilities, look for patterns, solve an equation, use direct reasoning, work backwards, solve a simpler problem, consider special cases first, use symmetry, use a model, etc. 
(3) Carry out the plan: This is about care in derivations, patience and avoiding mistakes. It requires persistence to stick with the plan chosen. If the plan clearly does not work, despite the best effort made, then it needs to be discarded and a heuristically second best plan needs to be chosen and pursued until a solution is found.

(4) Looking back: By taking time to analyse how the solution was found, and what worked and what didn't, valuable experience can be gathered for future use. This can help the development of an ability to feel what to do and use heuristic in future problem solving of the individual.

\subsection{Teaching of mathematics}

Polya in [14] has also commented on the principles of teaching. Though the most important duty of a teacher is to help the pupil to develop the ability of problem solving, this is complicated by the requirement that the pupil needs as much experience as possible in solving problems on their own, without any help. With absolutely no help, this may not however be possible, especially with very difficult problems. Facilitating the solution is best by hints and trying to keep the illusion of the pupils that they solve the problem themselves, as if the solution could have occurred to them too. Clearly, such methods are also important in order to be able accumulate technical knowledge within a short period of time.

A teacher's pedagogical view in [19] by Szvetits, who, during 40 years of teaching, made thousands of pupils like mathematics and also made them able to appreciate the beauty of problem solving, once stated that "... in my view among the many enablers of quality teaching, such as experience, skill and feeling, etc. there are three qualities, which stand out, and are more important than the rest: the teacher must know his subject well so that the pupils can clearly experience that and can benefit from it; the teacher should clearly enjoy mathematics so that his pupils can also feel that; and finally, the teacher should enjoy being among his pupils, which again must also be sensed by the pupils." These seemingly subjective statements, expressed as opinions, greatly contribute to our knowledge about effective methods of teaching problem solving. The same teacher trained and inspired nearly hundred pupils into winners of national and international mathematical olympiads from Hungary. 


\section{Competitive problem solving}

This section is split into two parts. the first examines the mental steps of the problem solver to find a solution. The second part is concerned with mental conditions of the problem solver outside the technical steps, which are purely psychological.

\subsection{Mental handling of solutions steps}

There are two psychological concepts we would like to use to describe problems solving: mental maps and heuristics. In this context a mental map is a set of relationships between mathematical objects kept in the mind, equivalently called a mathematical model. A drawing or set of equations is merely an extension of a purely mental map by physical aids to enable the use of the power of human vision to see relationships more clearly and simultaneously. Hence, using a piece of paper or computer screen, for visualisation, still remain within the scope of a mental map. On the other hand, heuristics is an intuitive, and largely subconscious, decision on the relevant options of transforming the mental map.

For our investigation, the main property of a mental map is that it is viewed like a "landscape". By this we mean that implications of relationships are quickly discovered based on known implication rules. This is not dissimilar from our ability to interpret a visual seen we are in, where we interpret relationships instantaneously. Hence we will coin here the term relationship-vision for the ability to discover one-step implications from a set of relationships in a mental map, based on sets of rules applicable and known the the problem solver.

For instance, in a geometric figure, relationship-vision could mean that we see how the size of some angles could be calculated from known ones or some ratio of sections could be equal to the ratio of others due to similarity. In derivation of equations, relationship-vision means that we can see what new equations could be derived from the current ones using simple operations. In number theory one would see, using relationship-vision, the factors that some of the terms must have, due to others having the same factors in an equation, etc. On the other hand, by its definition, relationship-vision is few-steps-only application of known rules. It does not imply that we "see" all the relationships derivable from a mind map/model of mathematical relationships.

Formally, application of a relationship-vision operator $R V[.$.$] to a set of$ relationships $R$ results in an extended set of relationships $R V[R] \supset R$. The relationship-vision operator $R V[.$.$] is however applicable to all three types of$ 
problems mentioned in the Introduction as (a)-(b)-(c) type of problems. The reason is that the existence, or otherwise, of some entries or relationships is usually concluded from a final relationship, which is derived in a sequence of relationshipvision steps. The solution to a problem is narrowed down by transformations of relationships which reduce the number of possibilities for the solution. We can assume, without any loss of generality, that some goal-relationship $R_{g}$, possibly expressed in higher order logic form, is to be proven, which leads to a solution of the problem, possibly by contradictio ad absurdum. Hence the mental operation of relationship-vision plays a fundamental role in the psychology of problem solving.

Application of steps of relationship-vision can result in different branches of an investigation tree, which has its roots at the assumptions of the problem and its branches are various relationship sets derived. The problem is to build up the tree, one of the branches of which will carry the "leaf" which represents $R_{g}$. The problem solver needs to decide which branch of the investigation it should choose to climb to get to the hypothetical leaf.

There are a number of factors that can influence the problem solver's decision on the direction they would take to arrive at a goal relationship:

(1) Forward heuristic step: The new set of relationships appears to be "nearer" to the goal relationship by some judgement of the problem solver. This means estimating the chance of success in a given direction by heuristics based on experience. We call this the heuristics operator $H E[. . / E]$ which produces a new set of relationships $H E\left[R / E_{H}\right]$ out of $R$ based on experience $E$ of the problem solver $H$.

(2) Backward heuristic step: The new set of relationships will imply the final relationship needed to be proved and hence the problem can be split into smaller parts. If the goal relationship is $R_{g}$ then

$$
D I V\left[R_{g} / E_{H}\right]=\left\{R_{1}, R_{2}, \ldots, R_{m}\right\} \rightarrow R_{g}
$$

This can also be called backwards derivation.

(3) Connective heuristic step: The new set of relationships formulate overall connections across the mental map and not just local ones, hence they are more suitable for the discovery of relevant overall relationships. The connectivity operator of deriving relationships is denoted by $C O N\left[R / E_{H}\right]$. Its use depends on the judgement and experience $E$ of problem solver $H$.

The problem solver's task is to connect the initial set of relationships $R_{0}$, which represent the assumptions of the problem, with $R_{g}$. 
The forward step, for instance, can include extending the geometric figure with some lines, circles or other geometric objects and their relationships to create a broader set of relationships. Another example of $H E\left[R / E_{H}\right]$ is a transformation step in a set of equations. The backward step $D I V\left[R_{g}\right]$ often means an analysis of the problem and splitting it into easier-to-prove parts. A connective step $C O N\left[R / E_{H}\right]$ can be crucial in cases where establishing local relationships in the mental model of the problem leads to nowhere near the solution as "global" relationships across the model are not grasped.

These three steps of mental operations are generally applicable and fit to most mental steps that a problem solver can carry out. Problem solving is a search process of finding forward $(1,3)$ and backwards (2) steps to get to a pathway from assumptions to the goal relationship. The unfortunate fact is that all three steps of types 1-3 heavily depend on the judgement as well as inspiration of the problem solver.

\subsection{Working conditions}

Psychology is also concerned with optimal conditions of making the right decisions. The problem solver can have a bad day when he or she is less effective. On the other hand, the examples of many successfully competing pupils show that, under the right set of methods of training in problem solving, dependency on environmental conditions can be reduced: history of competitions also show that there have been some consistently high performers.

Here we list a few observations of what is likely to be important for someone who is training to take part in mathematical competitions at high level.

(1) Teacher-pupil relationship. As pointed out in [19], robust conditioning of the mind to make it able to concentrate can be helped by steady and positive emotional attitudes enjoyed for a long period of time of problems solving, during which experience for heuristics is accumulated. Part of this process is stated in [14] in terms of the reasonably minimal help provided to the problem solver and facilitation of self-reliant work in problem solving exercises by pupils.

(2) Mental states during competitions. The series of relationship-vision steps, as described above, are most successful if the competitor is in a reasonably relaxed state. This can be achieved by enjoying problem solving even at the time of a competition. Such an attitude can conquer tiredness or ill disposition for health reasons. 
(3) The amount of problem solving during preparations. The less prior knowledge one has, the more one needs to rely on ingenuity at competition time. The less preparation is made, the more reliance will be placed on inspiration in terms of the investigation tree in heuristic steps. Best is hence to reduce uncertainties by accumulating a substantial amount of experience in problem solving exercises on hard problems. Depending on the level of competition, it is likely that a competitor needs to review all the problems which historically occurred in past competitions and perhaps much more to have any chance to get near to winning.

(4) Persistence of trying. It can often happen that pupils can spend hours on a problem from a past competition without producing solutions. Should then they be allowed to look up the solution? If the objective was only to find a solution one would say "yes". The objective is however not the solution but to learn how to solve problems. Dead ends need to be experienced. The urge to search for new avenues needs to be developed. Is it therefore perhaps better to put the problem aside for a while, sleep on it, and continue the next day? The advantage of doing this is that the investigative process will leave a strong trace in the cognitive processes, and hence in the ability of the problems solver. If repeated many times, their brains will find a way to solve problems of the same difficulty in a much shorter time period, eventually well within the time limits of competitions. Persistence brings the biggest rewards! This is certainly true for very advanced problem solvers; for beginners the teacher should provide a list of ideas and tips to try, but should never provide the solution.

(5) Self Confidence. If the discipline of persistence prevails, and the problem solver has solved a large number of problems, the arrival of a strong feel of self-confidence in one's ability to solve problems will eventually arrive and stay. Is self confidence in one's problem solving ability important? It is an invaluable power source of persistence and the source of the important belief at competitions that one can do it. The ability of strong focus is also supported by self-confidence.

(6) Is it by good luck to get inspired? Beginners of problem solving may think that by going through many possibilities it is random whether one finds the right idea and hence mainly good luck is needed to find the idea which leads to a solution or to be inspired. There is strong evidence against such an argument: there is a large number of young competitors who carried on winning national competitions and persistently performed at the top of the 
International Mathematical Olympiads. These pupils had brain mechanisms which made them always inspired, it was not a matter of being lucky.

It would however be wrong to conclude from this that winners of competitions would also solve any problem in areas of mathematics which are not usually the subject of competition problems, though they could build on their experience. One can safely only say that the evidence shows: for the type of problems which occur at secondary school competitions, it is possible to train gifted pupils to regularly perform at the top of competitions.

\subsection{The subconscious of discovering a solution}

Most of the above said has been concerned with the surface of the problem solving process, which are accessible by observing pupils and also by introspection. We have however limited knowledge on the workings of the subconscious and we can only speculate and derive from what is observable. We can perhaps state some conjectures:

A The subconscious plays an important role in the mechanisms of relationshipvision.

B There are some physiological/neural mechanisms which enable fast discovery of relationships, which can only be developed by experience of self-reliant problem solving.

C Heuristics, an inspired judgement on the direction one should proceed in an investigation, appears an even harder-to-develop subconscious process than relationship-vision. It could be hypothesized that the brain is able to analyse a lot combinations, and their likelihoods for success, in the subconscious.

In winners of International Mathematical Olympiads the subconscious process of $\mathrm{C}$ is likely to be particularly well developed beyond a strong ability and quickness in relationship-vision.

\section{Conclusions}

This paper has provided some observations on the psychology of mathematical problem solving. It has been pointed out that methods of meta-mathematics can convert problem solving into a well defined search-problem over steps of derivations by mathematical logic, starting from assumptions, axioms and known 
theorems. It has been concluded that problem solving is based on mental transformations of relationships in which methods of heuristics suggest directions for the investigations. The concept of "relationship-vision" has been introduced and three typical heuristic steps of problem solving have been identified. Psychology of problem solving has been commented on in terms of teacher-pupil relationships, mental states at competitions and best practice has been argued for when preparations are made. Finally, the role of the subconscious has been hypothesized in relation-vision and heuristics of problem solving.

\section{References}

[1] ADEPT: A Heuristic Program for Proving Theorems of Group Theory, 1966, http://hdl.handle.net/1721.1/6906.

[2] 7th International Joint Conference on Automated Reasoning, Concrete Mathematics, Addison-Wesley, July, 2014, http://cs.nyu.edu/ijcar2014.

[3] Special Issue on First Order Theorem Proving, Journal of Symbolic Computation 36, no. 1-2, (P. Baumgartner and H. Zhang, eds.) (2003), 1-286.

[4] W. W. Bledsoe, Splitting and reduction heuristics in automatic theorem proving, Artificial Intelligence 2, no. 1, 55-77.

[5] W. W. Bledsoe and P. Bruell, A man-machine theorem-proving system, Artificial Intelligence 5, no. 1 (1974), 51-72.

[6] 18 Unconventional Essays on the Nature of Mathematics, (R. Hersh, ed.), Springer Science, 2006, ISBN-10:0-387-25717-9, http://springeronline.com.

[7] J. Hadamard, The Psychology of Invention in the Mathematical Field, Dover Publications Inc. from Copyright of Princeton University Press, 1944.

[8] A. G. Hamilton, Numbers, Sets and Axioms: The Apparatus of Mathematics, Cambridge University Press, 1982.

[9] ZFC - Zermelo-Fraenkel Set Theory with the Axiom of Choice, in: Encyclopedia of Mathematics, (M. Hazewinkel, ed.), Springer, 2001, ISBN 978-1-55608-010-4.

[10] D. H. Jonassen, Toward a design theory of problem solving, Educational Technology Research and Development 48, no. 4 (2000), 63-85.

[11] P. Judea, Heuristics: Intelligent Search Strategies for Computer Problem Solving, Addison-Wesley, 1983.

[12] A. J. Nevin, A relaxation approach to splitting in an automatic theorem prover, Artificial Intelligence 6, no. 1 (1975), 25-39.

[13] W. Pohler, Proof Theory : The first step into impredicativity, 2003, http://wwwmath.uni-muenster.de/logik/Lehrveranstaltungen/04ss/LoSem/text.pdf.

[14] G. Polya, How to solve it, Princeton University Press, 1945.

[15] G. Polya, Mathematical Discovery, John Wiley \& Sons Inc., 1962. 
[16] L. Redei, Begründung der Euklidischen und Nichteuklidischen Geometrien, Hungarian Academic Press, Budapest, 1965.

[17] A. Robinson, Introduction to model theory and to the metamathematics of algebra, North-Holland, Amsterdam, 1963.

[18] A. H. Schoenfeld, Learning to think mathematically: Problem solving, metacognition and sense-making in mathematics, in: Handbook for Research on Mathematics Teaching and Learning, (D. Grouws, ed.), 1992, 334-370.

[19] Z. Szvetits, Together in the Fazekas - "Együtt a Fazekasban", in: Emlékezés Nagy Lászlóra - Remembering László Nagy, (J. Ungvári, ed.), Hajdú-Bihar Megyei Neveléstörténeti Egyesület, Debrecen, 1999, 40-41.

[20] Y. Wang and V. Chiew, On the cognitive process of human problem solving, Cognitive Systems Research 11 (2010), 81-92.

[21] Handbook of Control Systems Engineering, (L. C. Westphal, ed.), Springer, 2001.

SANDOR M. VERES

UNIVERSITY OF SHEFFIELD

SHEFFIELD

$\mathrm{UK}$

E-mail: s.veres@sheffield.ac.uk

(Received July, 2015) 